\section{(a) Cancer, Reproductive, Cardiovascular and Other Chronic Disease Prevention Program}

\section{What are our priorities?}

The National Institute for Occupational Safety and Health (NIOSH) Cancer, Reproductive, Cardiovascular and Other Chronic Disease Prevention (CRC) Program works with partners in industry, labor, trade associations, professional organizations, and academia. The program focuses on:

- Preventing and reducing incidence of adverse reproductive outcomes related to work

- Preventing and reducing incidence of occupational cardiovascular disease (CVD)

\section{What do we do?}

- Conduct research on occupational cancer on high priority worker populations and exposures and communicate findings.

- Conduct research on high-priority agents to identify and quantify risk of adverse reproductive health outcomes associated with workplace factors and communicate findings.

- Assess the association of workplace factors and exposures with sub-clinical/clinical CVD using a variety of methods.
- Preventing and reducing incidence of occupational cancer
- Create documents to assist stakeholders in CRC

- Promote use of CRC research by authoritative organizations such as the Occupational Safety and Health Administration, National Toxicology Program, International Agency for Research on Cancer, and National Fire Protection Agency.

- Maximize CRC resources by collaborating with external researchers to evaluate occupational risk factors in pre-existing health studies. prevention efforts.

\section{What have we accomplished?}

- Published the "NIOSH List of Antineoplastic and Other Hazardous Drugs in Healthcare Set-

- Generated information used by the Occupational Safety and Health Administration to support the revised silica standard and a new beryllium standard.

- Provided expert consultation on occupational hazards associated with cancer or reproductive effects to the National Toxicology Program; the International Agency for Research on Cancer; an international consortium conducting the International Nuclear Workers Study; and the International Association of Fire Fighters.

- Expanded work with the Nurses' Health Study to assess potential reproductive effects among healthcare workers exposed to antineoplastic drugs and high-level disinfectants. tings, 2016."

\section{At-A-Glance}

The Cancer, Reproductive, Cardio-

vascular and Other Chronic Disease

Prevention Program provides lead-

ership in preventing work-related

diseases related to many types of cancer,

reproductive health, and cardiovascular

diseases, as well as occupational

neurologic and renal disease. This

snapshot shows recent accomplishments

and upcoming work.

Percent of International Agency for Research on Cancer Evaluations citing CRC Program work

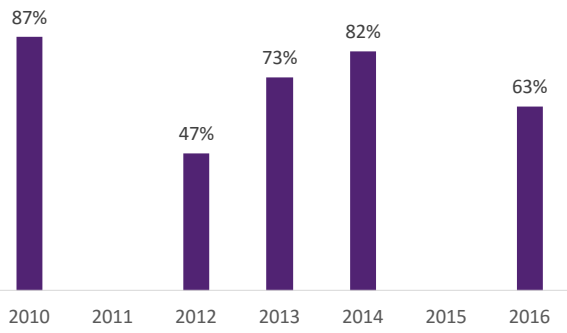

Source: International Agency for Research on Cancer (IARC) Note: No evaluations were published in 2011 and 2015; Includes only evaluations of occupationally relevant agents

- Provided research and provisional material lifting guidelines that were key components of clinical guidance statements adopted by the American College of Occupational and Environmental Medicine and American College of Obstetricians and Gynecologists.

- Published an industrywide biomonitoring evalvation of Bisphenol A (BPA) among manufacturing workers, finding that workers had elevated urine concentrations of BPA.

- Provided the scientific basis underlying proposed federal legislation to create a national fire fighter cancer registry. This legislation referenced a NIOSH cohort study of nearly 30,000 firefighters.

- Published "Current Intelligence Bulletin 68: NIOSH Chemical Carcinogen Policy."

\section{What's next?}

- Publish industrywide exposure assessment of:

- BPA among manufacturing workers, building on currently available information.

- Occupational exposure to flame retardant chemicals. A wide range of chemicals and mixtures are used as flame retardants; reported health effects have included liver and thyroid effects, developmental changes, and neurotoxicity.

- Publish translation of NIOSH Reproductive Health Topic Page in Spanish.
- Create multimedia training on occupationa risks for pregnant women doing lifting tasks.

- Publish NIOSH Science Blog on actions employers can take to improve workers' reproductive outcomes.

- Develop and share evidence-based recommendations for firefighters on preventing skin exposure to hazardous chemicals during building fires. place exposures and reproductive health

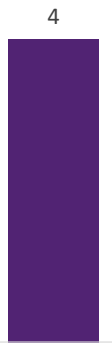

2015

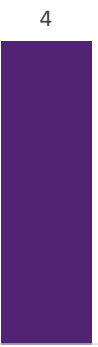

2016
Source: NIOSH Program Records

Publication Spotlight:

Current Intelligence Bulletin 68: NIOSH Chemical Carcinogen Policy

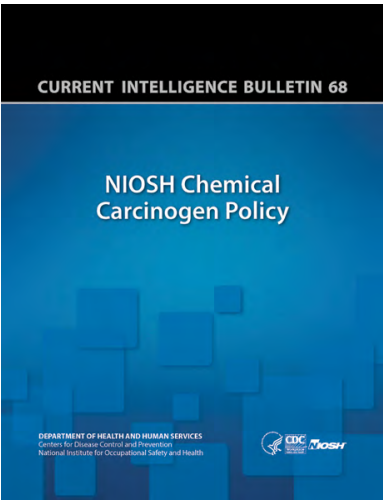

\section{Number of new associations between work-} outcomes evaluated by CRC Program research
DEPARTMENT OF HEALTH AND HUMAN SERVICES Centers for Disease Control and Prevention National Institute for Occupational Safety and Health
To learn more, visit

https://www.cdc.gov/niosh/programs/ crcd/default.html

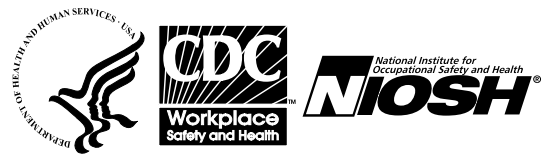

